\title{
Easier Said than Done: Methodological Challenges in Exploring Prevalence of Mental Health Illness among Rural Jail Inmates in the United States
}

\author{
Joseph Rukus \\ Assistant Professor \\ Department of Criminology, Sociology, and Geography \\ Arkansas State University \\ Jonesboro, Arkansas 72467 \\ Veena S. Kulkarni \\ Associate Professor \\ Department of Criminology, Sociology and Geography \\ PO Box 2410 \\ Arkansas State University \\ Jonesboro, Arkansas 72467
}

Corresponding author: Veena S. Kulkarni; vkulkarni@astate.edu, +1 870-972-3331

\begin{abstract}
A high prevalence of mental health illness among the incarcerated and has made jails one of the largest providers of mental health services, a purpose for which they are not equipped. Further research shows that failure to effectively treat mental health problems is a leading cause of post-release recidivism. Despite the evidence, there is no systematic investigation on the prevalence of mental health illness and its correlates in the context of rural jails. Our study looks at the methodological challenges of conducting this type of research in a rural jail environment. Inmates comprise a vulnerable population. Hence, the federally mandated regulations that are required to complete a study are greater, meaning Institutional Review Board approval is much more complex. In addition, there is a lack of familiarity with the process of collaborations on the part of researchers and jail staff. We outline how we addressed the challenges created by this dynamic. We found the proactive communication and ongoing dialogue amongst all parties to be a key element to addressing a variety of obstacles that arose in the process of taking our research from the drawing board into the field.
\end{abstract}

Keywords: human subjects, institutional review board, rural jails, mental health 
Research methods textbooks teach a detailed process designed to create the perfect study. They outline a series of painstaking steps that readers should undertake to create research protocols that are free of unwanted bias. While these are worthy goals to which all researchers should aspire, the stark reality of doing mental health research in a rural jail where budget and staffing constraints make such ideal methodologies a luxury. As Hammond (1967) details in his classic book Sociologists at Work: Essays on the Craft of Social Research, research in real world settings comes with a unique set of, challenges that are often compounded when interviewing inmates serving time in the county jail.

Yet, there is a dire need for such research because rural jails are often finding themselves on the front lines of an unrelenting addiction and mental health crisis. The Bureau of Justice Statistics reports that 64 percent of jail inmates have mental health problems (James \& Glaze, 2016) and that 26 percent of them experience serious psychological distress (Bronson \& Berzofsky, 2017). Approximately, 31 percent of all female and 15 percent of all male inmates have a serious mental illness such as major depressive order, bipolar disorder, and schizophreniform disorder (Steadman, Osher, Clark, Robbins, Case \& Samuels, 2009). Research indicates that failure to effectively treat mental health issues is a leading cause of post-release recidivism (Petersilia, 2003). A well-intentioned plan to close mental health facilities in the 1960s has morphed into public health catastrophe where jails and prisons are one of the country's largest providers of mental health services, a mission for which they are not equipped (AbuDagga, Wolfe, Carome, Phatdouang \& Torrey, 2016; Ford, 2015; Glasby \& Tew, 2015). While many major urban municipalities have been able to make investments which enable them to provide a minimal level of care (Cook County Sheriff's Office, 2017; San Francisco County Sheriff, 2014), rural communities lack both the resources and critical mass of inmates to duplicate such an infrastructure (Manfredi, Shupe \& Batki, 2015). As a result, mental health services in rural jails are often, at best, rudimentary.

The jails of Arkansas are not an exception (Petrimoulx, 2017), the problem being particularly acute in rural Arkansas. By virtue of necessity, caused by a shortage of mental health hospital beds, the region's jails have been forced to act as psychiatric treatment facilities, a mission for which they were not designed. There is lack of systematic information on the prevalence of mental health illness in jails and prisons. This dearth of research is particularly acute for rural jails which often lack inmate counts to get a sufficient sample size for quantitative analysis and are difficult to seek permission to access (Weisheit, Falcone, \& Wells, 2006). Studies on rural jail population of Arkansas are non-existent, let alone those that have an exclusive focus on the correlations and challenges pertaining to incarceration and mental health. The present study is being undertaken with the goal to fill this gap in our knowledge on the prevalence and correlates of the mental health illness among the inmate population in Arkansas jails. Our study sample comprise the jails in the rural counties in Arkansas. We anticipate the 
findings from our study to help design solutions targeted to alleviate the problem of mental illness in the rural criminal justice system.

While the need to understand mental health services in rural jails is undebatable, conducting studies that enable such investigation is hard. University institutional review boards require substantial safeguards be put in place to protect both inmates and researchers. These safeguards can be difficult to implement in rural jail environments. Doing successful research requires commitments and partnerships between all stakeholders, e.g. sheriffs, jailers, and university researchers that are radically different from those in more traditional research settings.

In this article, we discuss the challenges involved in doing this type of work and ways we addressed those challenges. We, by no means, suggest our methodologies are a definitive primer on the subject. However, we do believe we can offer potential solutions that can save future academics valuable time in doing any sort of inmate research in a rural context.

We organize the paper in the following way. First, we outline the context of our specific study. Second, we discuss the theory and the methodology that we employ. In the subsequent sections, we describe the challenges that we have come across at the various stages. Finally, we offer a few concluding observations.

\section{The Context of Arkansas: The Two Study Counties}

Given that the U.S. Census Bureau classifies both study counties as rural, the criminal justice system is plagued by funding constraints that is typical of the rural areas nationally. It is, therefore, not surprising that in response to the crisis posed by inmates experiencing mental illness, the facilities in both the counties resort to ad hoc means to tackle the problem.

While these interventions may be successful in preventing mentally ill inmates from harming themselves or others, they represent only a short-term fix to what, in most cases, is a long-term systemic problem. Additionally, the relatively (to other states) higher prevalence of mental illness and lower access to care in Arkansas (Mental Health America, 2016) exacerbates the situation. In the study region, there is only one acute care facility, and it has only 30 beds. Often high occupancy and limits on the type of patients that can be handled means inmates suffering from severe episodes are frequently transported to a far-away city for care. Such transportation implies that a deputy needs to be taken off regular duty or paid overtime, both costly propositions. Hence, the state's resource deficit further strains already thinly-stretched county law enforcement's ability to respond to the mentally ill.

While the prevalence of mental health illnesses among the incarcerated population is undisputed, there is no systematic study examining the extent, nature and its correlates in the 
context of rural jails generally, and in Arkansas in particular. Our goal was to conduct a study that examined the extent and the correlates of the prevalence of mental health illness among the incarcerated in the study jails. In the process, we would help fill a significant void in the literature. Creating a methodology to achieve our goal required starting from a nearly blank slate. Few resources exist detailing how to do research in rural jails. Most studies concentrate on prisons as opposed to jails since inmates are incarcerated longer, facilitating longitudinal studies, and larger prison populations make it is easier to obtain an optimal sample size for statistical analysis (Cook, 2009). Also, the limited extant studies tend to be overwhelmingly focused in larger urban areas, not in rural communities (Wells and Weisheit, 2004). Hence, the findings we share in this paper potentially have national implications on conducting methodical investigations in rural areas.

\section{Methodology}

Our study sites involved two jails in rural Arkansas (Figure 1). One jail had approximately 100 beds while the other had approximately 300 beds. Neither jail had previously been involved with any sort of university research study. The sheriffs of both the counties are aware of the problem and willingly agreed to the research because they were looking for information that would help them better address the problem.

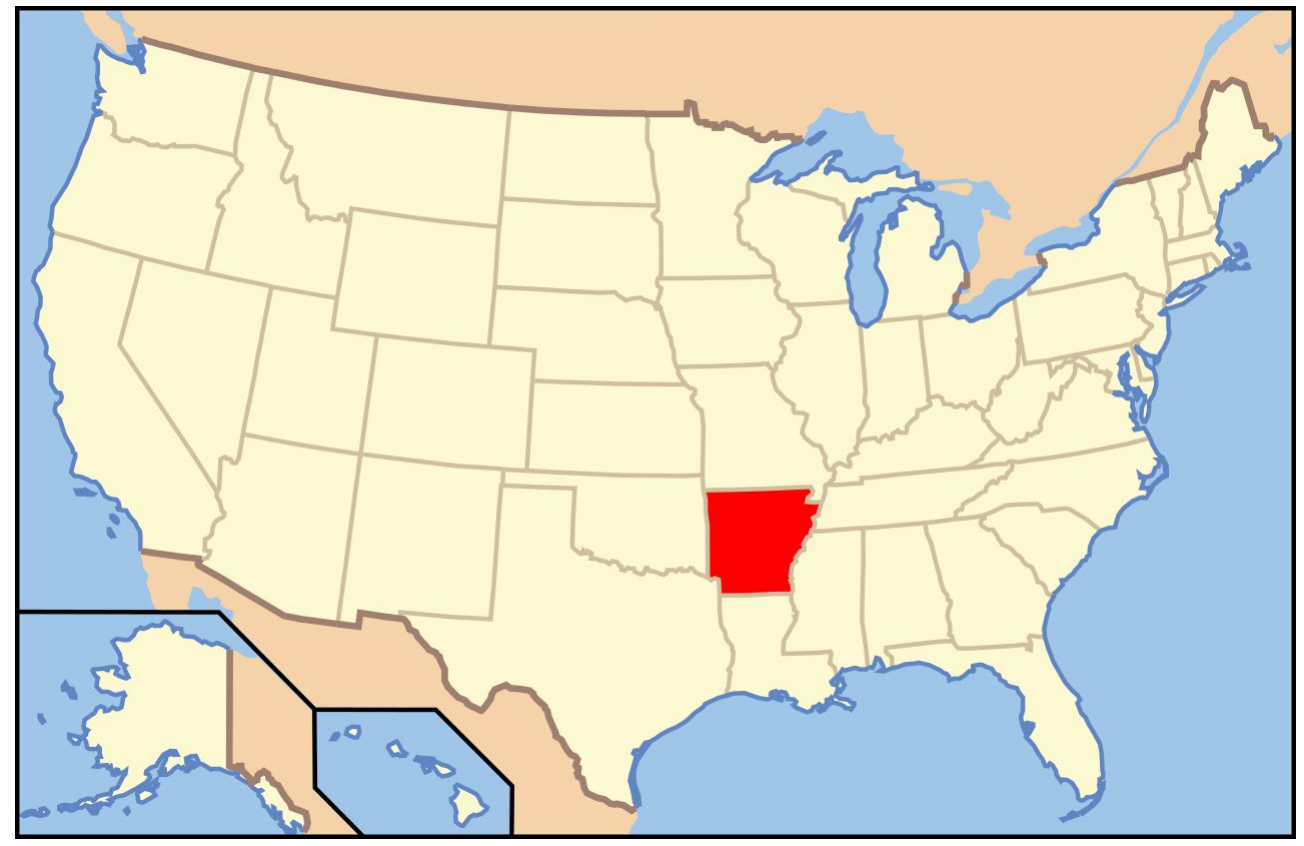

Figure 1: Location of Arkansas in the United States

(https://en.wikipedia.org/wiki/File:Map_of_USA_AR.svg) 
We employed a two-pronged methodological design comprising of quantitative and qualitative components or what is also known as mixed-methods approach. The mixed-methods approach is found to be especially advantageous when investigating complex social contexts such as violence and addiction (Testa, Livingston \& VanZile-Tamsen, 2011). In-depth interviews with open-ended questions help capture insights that get missed in quantitative surveys that comprise close-ended questions with finite options based on the extant literature. The two techniques complement each other as qualitative interviews provided deeper context to quantitative results. We choose this multi-layered approach because of the complexity involved with studying addiction and mental illness through the lens of the criminal justice system.

For the quantitative component we relied on the jail records to gather data on the two dependent variables, namely number of disciplinary reports and number of arrests. While our independent variable comprised the existence of mental health issues, the control variables included characteristics such as age, race, marital status level of education, occupation. We compared the arrest records of inmates with mental illness to inmates without mental illness.

The qualitative part comprised interviewing deputies, jail staff and an important stakeholder group frequently ignored in jail research, inmates. The in-depth interviews aimed to capture the nature and severity of the mental health issues and seek suggestions on possible interventions which could improve the situation. We interviewed both mentally and nonmentally ill inmates. To best document the nature of the need, we wanted to "know what we didn't know" and we believed the qualitative interviews provide us with that data. The study was completed in July 2018.

The theme of the present paper is to delineate the methodological challenges in executing the study. We find that challenges we face are a mix of those arising from a) the study sites located in rural regions and b) the study group being a vulnerable population. We present our experience as a case study. Case studies are a useful technique to explore, describe and/or explain an issue that has relevance across space and time by using a specific case (Yin, 2014). Hence, the findings from case studies, albeit not generalizable in a manner similar to a large sample quantitative inquiry, are potentially informative when conducting studies on comparable subjects and settings.

In an ideal scenario, theory and methodology should directly inform each other, regardless of whether the research is deductive or inductive. Yet investigations concerning two vulnerable groups, inmates and individuals with mental illness, involve safeguards which make using ideal methodologies extremely difficult. Additionally, such inquiries rarely engage university researchers. This lack of familiarity on part of both jail officials and academics makes the task of studying rural jails more onerous. 
Figure 2 conceptualizes the central actors in the study. We categorize the university and within the university agencies such as the Institutional Review Board (IRB) that are integral to the university as institutional. The administrative component includes the county jails. As will also be noted further in the paper, both the institutional and administrative actors had to interact with outside service providers to allows the study to take place. In the ensuing paragraphs, we describe the hurdles we experienced in coordinating amongst the various actors.

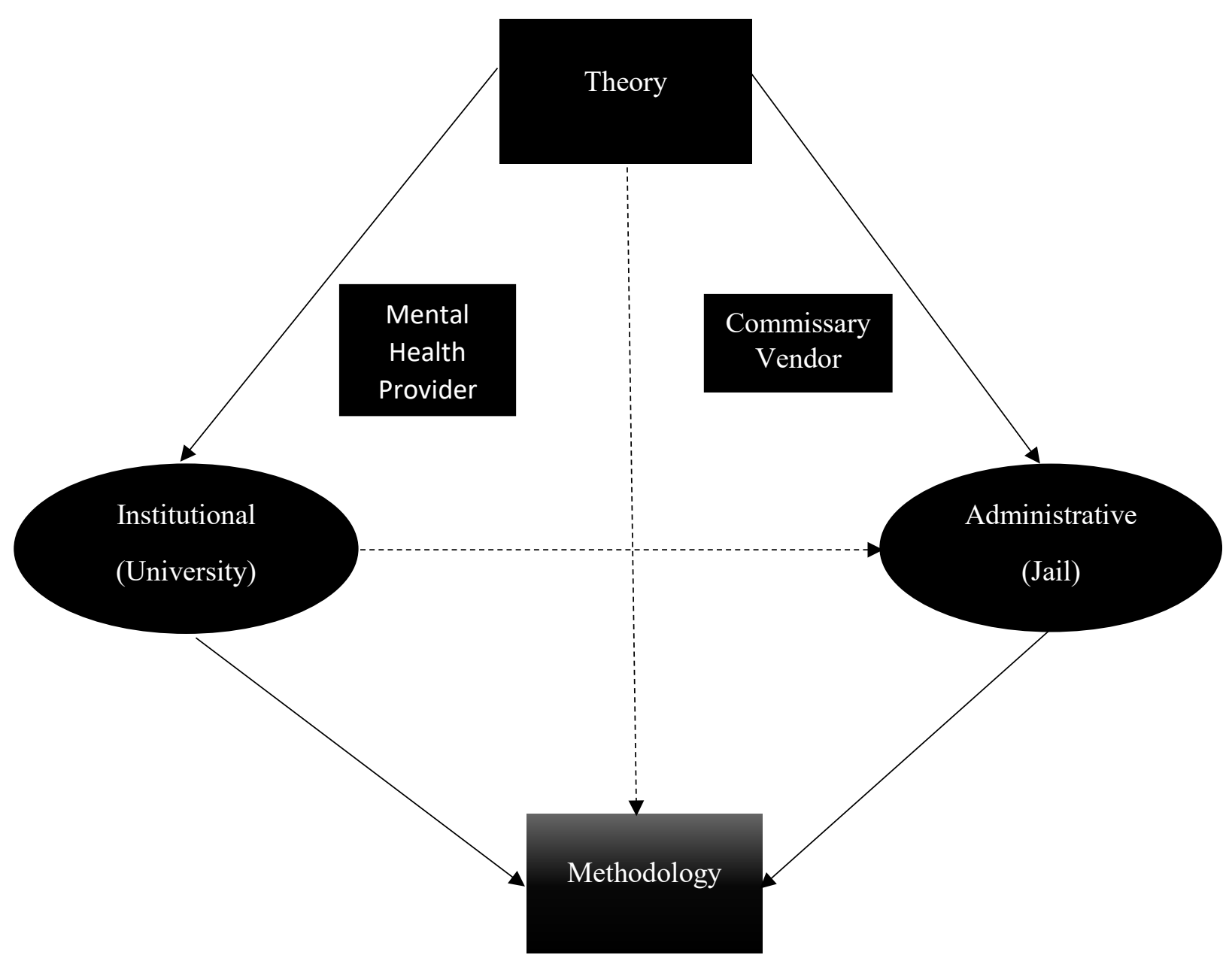

Figure 2: Central Actors in Conducting Research on Prevalence of Mental Health Problems Amongst Inmates in Rural Jails 
Easier Said Than Done: Methodological Challenges in Exploring Prevalence of Mental Health Illness among Rural Jail Inmates in the United States - Rukus and Kulkarni

\section{Challenge 1: Institutional Capacity}

While all research that involves human subjects require federally mandated regulations to be followed, the safeguards are more stringent when studying, amongst others, groups such as minor children, mentally ill people, and inmates. Hence, the fulfillment of the three certain basic ethical principles laid out in the Belmont Report for the protection of human subjects, namely 'respect for persons', 'beneficence' and 'justice' (National Commission for the Protection of Human Subjects of Biomedical and Behavioral Research, 1978) requires more steps to be undertaken to make the study compliant. It is therefore not surprising that when dealing with a study such as the one we are doing, the IRB, an agency that every university houses to review research projects, is overly cautious. At our university, the concerns were compounded by the fact the IRB had never approved a study involving inmate research in the past. For the IRB members, this was an uncharted territory and that implied that there was no infrastructure in place to guide them.

Given that one the PIs had previously designed and conducted inmate research, we were not completely unaware of the plausible difficulties in obtaining the IRB approval. We decided our best approach was to partner with the IRB from the beginning of the process. We wanted to be certain that they would not be surprised by any part of the protocol when it came time for review. Rather than presenting the study and wait for feedback from the meeting of the IRB members, we worked with our IRB officials throughout the design process. It helped us address the concerns as they arose and have procedures in place to alleviate items that the IRB would want addressed at the review.

One of those concerns, and a major one, was regarding confidentiality of the inmates. Our protocol entailed a stipend payment of $\$ 20$ commissary credit to inmates for participating in interviews. IRB had serious concerns about how the payments would be made while keeping inmate identities secret. Complicating these efforts was the need on part of the university accounting to accurately track how funds were being spent. We initially thought the confidentiality issue could be easily addressed by using the department procurement card to purchase commissary credits via the commissary vendor's website. However, when we tried to work out a procedure to accomplish this, we discovered that state credit cards were prohibited from receiving approval codes for purchases made with commissary vendors (Arkansas Department of Finance and Administration, 2017). The prohibition was to prevent potential payments by state employees to inmates for nefarious activities.

The situation created a dilemma. At this point, we contacted the IRB to inform them of the problem. We came up with a few possible solutions each of which had its pros and cons. The first was to petition that state to allow an exception to be made for our procurement card or issue a special procurement card for the study. While this would have created an easy audit trail, the 
time involved would have been substantial and would have severely delayed the study with no certainty of success. The second option comprised getting a cash advance from the university and using the money to purchase money orders that would be given to the commissary vendor to issue credits. However, university rules required documentation and that documentation, namely the money orders, would have the name and inmate number of the interviewee violating confidentiality.

We worked with the IRB to come up with a third option which proved to be our solution. The university issued a check to the commissary vendor for the stipends. Subsequently, the commissary vendor credited money to inmate accounts once interviews were completed and that meant a direct involvement with the commissary vendor in the process. One of the positive aspects of working with local businesses in rural areas is their nimbleness in responding to unusual situations. We contacted the commissary vendor who informed us they did not have a procedure in place to put money into a master account which would then be gradually distributed into individual inmate accounts but realized the importance of the project. They agreed to manually process the stipends outside of their normal system. In addition, they substantially cut their service fee to $\$ 1.00$ per stipend, which they estimated was their direct costs, not including staff time which they donated, to process the stipends. Once informed of their agreement, the IRB worked with the department in the university that oversees external (to the university) funds to create a memorandum of understanding that the IRB members would find satisfactory.

This spirit of partnership with the IRB continued throughout the approval process. At one point, the IRB was unsure of how to correctly apply a regulation to an element of our protocol. We expressed our understanding of the regulation but also suggested they contact an IRB inmate representative that one of the PIs had worked previously at another university which had extensive experience approving inmate research. They contacted the representative who gave valuable advice as to how they applied the regulations in question and consulted with our IRB, as needed, throughout the approval process.

The above is an illustration of how the IRB procedures and regulations can be tedious. As a result, the relationship between researchers and IRBs often becomes adversarial and reactive. We found that a proactive approach that involves working with IRB from the beginning yielded better results for all parties involved. It sped up the approval process because issues were addressed at onset and the IRB felt more secure in approving the study because their input had been incorporated throughout the process. It was a true example of a win-win.

While our partnership with the IRB eliminated many concerns before our protocol went to the full board, there were still issues expressed by board members. Most of the revisions recommended by the IRB members were minor modifications to the protocol or included changing verbiage in our notice of informed consent, but a major concern arose. It pertained to 
our gathering information from inmates on their experiences with jail mental health services. We believed that self-reporting on mental health conditions and jail mental health processes posed minimal risk to the inmates. However, one of the IRB members however raised objection and believed more mental health resources were needed. The member requested that we either add a third PI with PhD in psychology or provide 24-hour access to a mental health care provider for the entire duration of the study.

Both the alternatives appeared infeasible. Gaining access to the jails had involved a multiyear process in which the PIs and one of the sheriffs had worked closely together on other projects. Our work together had allowed us to build a mutual level of trust, a critical component to doing inmate research. Introducing a third party that neither the PIs or the sheriffs knew would introduce an unknown element that could complicate matters. We were all concerned about the ramifications of bringing in a third party when the protocol approval was so far along. Additionally, 24-hour access to a mental health professional would be an expensive proposition and was well beyond the study's budget.

Faced with an IRB request that would have effectively and extremely delayed or even stopped the study outright, we decided to pursue a different track. We contacted the mental health provider contracted by both jails for evaluations and emergency responses and asked them to evaluate our protocol and questionnaire. They agreed with our initial assessment that risk posed to inmates by our study were extremely minimal. We asked them if they would be willing to work with us to craft a compromise. At first, the provider was hesitant because they were afraid they would be placed in a position where they would be providing uncompensated treatment for severe mental health conditions that had not been caused by the study. After a lengthy discussion, we devised an alternative where a psychologist would be available to receive calls from study participants at certain time intervals. The psychologist would then do an evaluation over the phone and would activate standard jail and provider protocols if inmates were in crisis. As the solution was based on the mental health care provider's professional assessment of the need, the majority of the IRB members were satisfied with the compromise. The IRB approved our proposed solution. The IRB, the mental health provider, and the department dealing with external grants worked with one another to craft a memorandum of understanding that met all the regulatory requirements.

Another potential issue arose with the inclusion of the contact phone number of the IRB on the notice of informed consent. While the purpose of the number was to address concerns about the rights of participants, based on the previous experience of one of the PIs, that phone number was likely to be used for resolving logistic and other matters related to the payment of the stipends. In a prior instance, inmates had made claims about not receiving stipends even though the stipends had been processed and credited to their accounts. If repeated, this would put the IRB in the role of providing customer service, a task that they are unaccustomed to perform. The 
IRB and we, the PIs, mutually agreed that all requests would be forwarded to us for handling. As one would expect, kinks had to be worked by the commissary vendor in manually processing the stipends. For instance, when a change in the vendor's computer systems delayed stipend processing, the inmates contacted us. There were also cases when the inmates called the IRB when we were not available owing to other professional obligations. As we had decided, the IRB informed us of the call and we handled the situations accordingly. The lesson learned from this instance is that an ounce of prevention, warning the IRB and coming up with handling procedures in advance, was worth a pound of cure. Additionally, the above underscores the importance of working the IRB as partners as opposed to viewing them as an adversary.

An intense IRB process is an absolute necessity when working in a jail environment because the potential for risk is real. However, this process need not be one that is adversarial. Working with IRB as a partner allowed both parties in the review process to know the goals and concerns of the other and facilitated both working jointly to craft solutions. Overall, our partnership with the IRB resulted in a protocol that was much stronger from a research perspective and added a number of different levels of safeguards which greatly increased the safety for all parties involved.

\section{Challenge II: At Rural Jails}

We also faced challenges at the jail. Similar to the IRB, we worked hard to develop a partnership with jail staff to address concerns. However, this process was more delicate than that with the IRB. Jail operations are not always smooth and the jail staff frequently needed to come up with quick solutions to problems that suddenly present themselves. Jail staff were afraid that their actions might come under unnecessary scrutiny. This is a syndrome which can be referred to as "Here come the PhDs who want to change everything." This syndrome can be exaggerated in rural jail settings where staff often have no experience working with university-based researchers. Our two study sites were no exception in this respect. To counter this perception, we had meetings with all critical staff to come up with appropriate procedures. While they did not question our motives, we decided to be proactive and make it very clear that our goal was to look at the jail's response in a macro sense and situate it as a structural issue, and not to place blame or scrutinize the actions of individual staff members. We also expressed empathy with them about the fact that they were doing a very difficult job in less than ideal circumstances. We noted several times that one goal of our research was to find ways of working with mental health illness that would make their jobs easier in the long run. While this did not allay all suspicions, we found that it went a long way in gaining the trust we needed to complete the study.

One area of conflict was the perception of the threat posed by inmates. By their very nature, jailers are not accustomed to viewing inmates as vulnerable populations that require extensive safeguards to protect their confidentiality. As a result, there was resistance to some of 
our procedures. Jailers feared leaving us alone with inmates and strongly requested that an officer be present at the door or in the room for the duration of the interview. Such a procedure would have gone against the IRB regulations. As such, we worked to come up with a solution that would satisfy IRB protections. To assure we received a cross section of inmates, we selected inmates based on a random number that we applied to inmate identification numbers. We set up a system where we were issued radios. We used them to inform jailers to bring us our next potential interviewee and notify them when interviews were complete.

Further, we added a safeguard to our protocol that would give an inmate the option of staying with us for 30 minutes in the interview room if they chose not to participate, so officers would not have the knowledge of whether they had participated in the interview. While hesitant to leave us alone with inmates, we reached a compromise where inmates would remain handcuffed for the duration of the interview, not ideal but acceptable. In addition, we agreed to have no more than one pen open in the room and agreed to remove staples from the copy of the informed consent that inmates received. We also agreed to modify the procedure if safety issues arose. We are happy to report no safety concerns arose.

Collecting the quantitative data had its own set of challenges. Initially, we had hoped that we could download data into a spreadsheet which we could load into statistical software for analysis. Since the information we needed was stored in different parts of the jail computer system, this proved implausible. As a result, we needed to manually collect data. We developed a sheet and a graduate assistant collected the data off the jail computer. Another graduate assistant then entered the data into a spreadsheet which we then loaded into the computer for analysis. Finally, a letter notifying each inmate that research data had been collected, along with contact information for questions, was placed in each inmate's jail file. As our sample consisted of 954 observations, this was much more time consuming then initially expected. Further complicating and slowing the process down was the fact that each jail asked different questions and stored the information differently, requiring the PIs to do a bit of investigation to put questions on the sheet that could be applied to data in each jails' computer systems.

This procedure encountered an unexpected hurdle. Manually collecting data from the jail's computer system meant that we needed access to open computers. As office space is at a premium in rural jails, the graduate assistant working on the computer needed a great deal of flexibility and had to schedule time in advance when there would be open office space.

In addition, a high degree of flexibility was required for doing inmate interviews. In our first study site, a courtroom is connected to jail to limit the need for inmate transport outside the facility, an arrangement that is common in many rural communities. While this increases efficiency, it also creates a lot of movement inside the jail as inmates are escorted to and from their court appearances. Justifiably, jail staff did not want the additional complication of 
transporting inmates to and from our interview room while inmates were being escorted to and from the courtroom, meaning we had to schedule around planned court times. However, this was not always enough because, at times, court went long. When this occurred, we had no choice but to reschedule our interview dates.

Another scheduling issue occurred because our interview room served a variety of purposes. It was used to conduct assessments by the mental health provider and was also used by a local religious group that was doing addiction outreach. Predicting the times these functions would end proved more of an art than science meaning we often had downtime in the jail where we had to wait for the room to become available, a frustrating experience for both the jailers and the research team. We mention this to caution fellow researchers that the best laid plans frequently go awry when conducting this type of research and that a substantial amount of patience and flexibility is required.

By their very nature, jails are unpredictable environments where situations can evolve and devolve rapidly. The main lesson from our experience is that a great deal of flexibility is required when using the jail as a research site. As with the IRB, an ongoing dialogue addressing goals and concerns is essential. One added benefit of this open communication was that jailers were able to let us know their experiences in dealing the issues addressed in our study. This knowledge opened our eyes to facets of the problem which we had not previously considered. Overall, the level of cooperation created a richer study and allowed us to collect data which were more relevant to specific challenges faced by each individual facility.

\section{Conclusion}

Our goal in narrating our experience is to provide information to our peers pursuing inmate research in rural jails. While undertaking these kind of studies, the path between theory and methodology often needs to take a detour to handle both the needs of protecting participants through the IRB process and the needs of the jail. Addressing these needs can be a complicated juggling act. We hope discussing the hurdles we faced and detailing how we dealt with them is of assistance to fellow researchers and will help them as they create their protocols.

One of the key lessons we learned was the value of partnering with all parties involved. Realizing the issues faced by each of the parties and addressing them as the study progresses is key to success. For instance, working closely with the IRB from the beginning helped overcome many of the concerns that the IRB had. The important part of this partnership is that there are no surprises. We presented our study goals and the methodology we felt best accomplished those goals and then worked closely with the board to create a protocol that would pass muster with the full board. 
Part of this process involved working closely with the other actors. One advantage to doing research in a rural environment is that other actors in the process are more likely accustomed to coming together to accomplish a common goal. For example, our commissary vendor realized the value of our exploration and altered their procedures to our needs, something which would be much more difficult in a large, urban setting. We find that there was a similar willingness to cooperate with both the local mental health service provider and jail staff. Communication and respect are critical components of the process and, in rural settings, trust plays a major role in many interactions.

Also, we would like to underscore the need for patience. Even working with the IRB to create a protocol, the process of getting a study approved involving inmates is usually a lengthy process. Researchers should be prepared to make revisions to their methodologies to assure the study meets regulations governing inmate research. Additionally, jails have situations that arise which can make it difficult to predict when there is staff availability and when the facility is capable of doing interviews. There are times when a planned interview day will need to be rescheduled due to constraints of the facility at the planned time of the interviews. A friendly and personable disposition goes a long way to alleviate the stress such situations can cause.

Putting all the methodological and logistic pieces in place to do inmate research is difficult, especially given that a mixed -methods approach is more desirable when investigating complex and sensitive topics such as criminal behavior and mental health. Conversations with inmates allow investigators to get a level of depth, which can explain quantitative data in a way that cannot be matched by using control variables. Their insights provide rich data that cannot be obtained from any other source. Most agree that the current process of using the criminal justice system as a mechanism to deal with failures in the mental health care system is unsustainable. Inmates can provide information that can be vital to solving this crisis. Our hope is our experience helps other researchers tap this valuable source of information.

Like all studies, our case study has limitations. By its very nature, a case study provides information on only one case and therefore has limited generalizability. Every university and every jail is different. As such, there is not a one size fits all approach that works. Nonetheless, the value of knowing the challenges posed by in communicating and seeking cooperation with institutions, inmates and law enforcement agencies in specific instances cannot be dismissed.

As for future research, we firmly believe that other researchers should be encouraged to publish their own experiences in doing rural inmate research. Given that studies exclusively dedicated to describing methodology are few and far between, the information on the nitty gritty of implementing studies to examine rural criminal justice system is scant. A greater dissemination of methodological challenges and the practices adopted to counter them would be 
helpful in devising more fruitful techniques to conduct research in the criminal justice system located in the rural regions.

\section{References}

AbuDagga, A., Wolfe, S., Carome, M., Phatdouang, A., and Torrey, E. (2016). Individuals with serious mental illnesses in county jails. Washington, DC: Public Citizen.

Arkansas Department of Finance and Administration (2017). Procurement. Retrieved from https://www.dfa.arkansas.gov/procurement.

Bronson, J. and Berzofsky, M. (2017). Indicators of mental health problems reported by prisoners and jail inmates, 2011-2012. Washington, DC: Bureau of Justice Statistics.

Cook County Sheriff's Office (2017). Office of mental policy and advocacy. Retrieved from https://www.cookcountysheriff.org/departments/mental-health-policy-advocacy/.

Cook, C. (2009). Jail staff professional orientation \& attitudes towards victimization. $\mathrm{PhD}$ dissertation. Gainesville, FL: University of Florida.

Hammond, P. (1967). Sociologists at work: Essays on the craft of social research. New York, NY: Anchor Books.

Glasby, J. and Tew, J. (2015). Mental health policy and practice. (3 ${ }^{\text {rd }}$ edition). New York, NY: MacMillan.

Ford, M (2015, June 8). America's largest mental hospital is a jail. The Atlantic Monthly. Retrieved from https://www.theatlantic.com/politics/archive/2015/06/americas-largestmental-hospital-is-a-jail/395012/.

James, D. and Glaze, L. (2016). Mental health problems of prison and jail inmates. Washington, DC: Bureau of Justice Statistics.

Manfredi, L., Shupe, J., and Batki, S. (2005). Rural jail telepsychiatry: A pilot feasibility study. Telemedicine and E-health, 11(5), 574-577. https://oi.org/10.1089/tmj.2005.11.574

Mental Health America (2016). 2016 state of mental health in America - Ranking the states. Retrieved from http://www.mentalhealthamerica.net/issues/2016-state-mental-healthamerica-ranking-states. 
National Commission for the Protection of Human Subjects of Biomedical and Behavioral Research. (1978). The Belmont report: Ethical principles and guidelines for the protection of human subjects of research. [Bethesda, Md.]: The Commission.

Petersilia, J (2003). When prisoners come home: Parole and prisoner reentry. New York, NY: Oxford.

Petrimoulx, D. (2017). Mental illness causing crisis in AR jails. KARK 4. Retrieved from http://www.arkansasmatters.com/news/local-news/mental-illness-causing-crisis-in-arjails/442954240.

San Francisco County Sheriff (2014). Jail health services: Behavioral health and reentry program. Retrieved from: http://www.sfsheriff.com/files/BehavioralHealthReentryProgramDescription.pdf.

Steadman, H., Osher, F., Clark Robbins, P., Case, B. and Samuels, S (2009). Prevalence of serious mental illness among jail inmates. Psychiatric Services, 60(6), 761-765. https://doi.org/10.1176/ps.2009.60.6.761

Testa, M., Livingston, J. A. and VanZile-Tamsen, C. (2011). Advancing the study of violence against women using mixed methods: Integrating qualitative methods into a quantitative research program. Violence Against Women,17(2), 236-250. https://doi.org/10.1177/1077801210397744

Weisheit, R., Falcone, D., and Wells, L. (2006). Crime and policing in rural and small-town America. ( ${ }^{\text {rd }}$ edition). Long Grove, IL: Waveland Press.

Wells, E.L., and Weisheit, R.A. (2004). Patterns of urban and rural crime: A county level comparison. Criminal Justice Review, 29(1), 1-19 https://doi.org/10.1177/073401680402900103

Yin, Robert K. (2014). Case study research: Design and Methods. (5 edition). Thousand Oaks, CA: SAGE Publications, Inc. 